\title{
PACKING DIMENSION OF IMAGES OF ADDITIVE LÉVY PROCESSES
}

\author{
LIANG ZHANG
}

\begin{abstract}
In this paper we generalize the packing dimension profiles defined by Khoshnevisan, Schilling, and Xiao (2012) to higher dimensions and use it to compute the packing dimension of an arbitrary image of an additive Lévy process.
\end{abstract}

\section{INTRODUCTION}

An $N$-parameter random field $X=\left\{X(t): t \in \mathbb{R}_{+}^{N}\right\}$ on $\mathbb{R}^{d}$ is an additive Lévy process if each sample path of $X$ satisfies:

$$
X(t)=X_{1}\left(t_{1}\right)+\cdots+X_{N}\left(t_{N}\right) \quad \forall t=\left(t_{1}, \ldots, t_{N}\right) \in \mathbb{R}_{+}^{N},
$$

where $X_{1}, \ldots, X_{N}$ are independent one-parameter Lévy processes on $\mathbb{R}^{d}$. Let $\Psi_{1}, \ldots, \Psi_{N}$ denote the Lévy exponents of $X_{1}, \ldots, X_{N}$, respectively. Then the characteristic function of $X$ is $\mathbb{E}\left[e^{i \xi \cdot X(t)}\right]=\exp \left(-\sum_{j=1}^{N} t_{j} \Psi_{j}(\xi)\right)$, for all $\xi \in \mathbb{R}^{d}$.

Additive Lévy processes have been used to study Lévy sheets as well as the intersection and self-intersection of Lévy processes. For example, see Dalang and Walsh [1, 2], Fitzsimmons and Salisbury [5], Kendall [7, and Khoshnevisan and Xiao [10, to cite some of the references.

In this paper we compute the packing dimension of the images of an arbitrary additive Lévy process. For a nonrandom Borel set $F \subseteq \mathbb{R}_{+}^{N}$, the random image $X(F)$ usually exhibits fractal structure, and it is natural to calculate the Hausdorff dimension and packing dimension of $X(F)$. (See Falconer [3] for definitions of Hausdorff and packing dimension.) When $N=1$, there is a rich literature for computing the Hausdorff dimension $\operatorname{dim}_{\mathrm{H}} X(F)$ of $X(F)$; see Khoshnevisan and Xiao [11] and its extensive reference. On the other hand, there have been few results about the packing dimension $\operatorname{dim}_{\mathrm{P}} X(F)$ of $X(F)$. In a recent paper, Khoshnevisan, Schilling, and Xiao [9] defined a new family of packing dimension profiles $\operatorname{Dim}_{\kappa}$ and showed that

$$
\operatorname{dim}_{\mathrm{P}} X(F)=\operatorname{Dim}_{\kappa} F \quad \text { a.s. }
$$

When $N>1$, a formula for the Hausdorff dimension $\operatorname{dim}_{\mathrm{H}}(F)$ was given by Yang [16. However, there is little known about the packing dimension $\operatorname{dim}_{\mathrm{P}} X(F)$.

The packing dimension profiles defined in [9] are probabilistic but have analytic significance as well. They can be regarded as an extension of the packing dimension profiles defined by Falconer and Howroyd [4] and Howroyd [6]. However this extension is restricted to subsets of $\mathbb{R}$ while the dimension profiles of [4] and [6] are defined for subsets of $\mathbb{R}^{N}$ for all $N \geq 1$. It is natural to ask whether we can

Received by the editors June 18, 2012 and, in revised form, September 19, 2012.

2010 Mathematics Subject Classification. Primary 60G17; Secondary 60G60, 60J45, 28A80. 
generalize the dimension profiles of $\left[9\right.$ to subsets of $\mathbb{R}^{N}$ for $N>1$ so that the new dimension profiles extend that of $[4]$ and $[6]$.

In this paper, we generalize the packing dimension profiles defined in 9] to higher dimensions and derive a multiparameter version of (1.2). We also show that our definition gives probabilistic interpretation to the packing dimension profiles of Falconer and Howroyd [4] and Howroyd [6]. The extension from $N=1$ to $N \geq 1$ is not straightforward since the structure of $\mathbb{R}^{N}$ is richer than $\mathbb{R}$ if $N>1$. In particular, the main theorem of 9 relies on a stopping time argument, while we cannot define stopping times for general multiparameter processes. In order to overcome this difficulty, we employ the potential theory for additive Lévy processes that was developed in Khoshnevisan, Xiao, and Zhong [14.

The rest of this paper is organized as follows. In Section 2 we define a family of box dimension profiles and packing dimension profiles, and present the main result. In Section 3, we prove our main result. Finally, we give some applications in Section 4

\section{Dimension profiles}

2.1. Box dimension profiles. Recall that $X=\left\{X(t): t \in \mathbb{R}_{+}^{N}\right\}$ is an additive Lévy process on $\mathbb{R}^{d}$. Define the associated process $\widetilde{X}=\left\{\widetilde{X}(t): t \in \mathbb{R}^{N}\right\}$ by

$$
\widetilde{X}(t):=\sum_{j=1}^{N} \operatorname{sgn}\left(t_{j}\right) X_{j}\left(\left|t_{j}\right|\right) \quad \forall t \in \mathbb{R}^{N} .
$$

It follows that

$$
\forall s, t \in \mathbb{R}_{+}^{N}: \tilde{X}(t-s) \text { has the same distribution as } X(t)-X(s),
$$

and the Fourier transform of $\widetilde{X}(t)$ is given by

$$
\mathbb{E}\left[e^{i \xi \cdot \widetilde{X}(t)}\right]=\exp \left(-\sum_{j=1}^{N}\left|t_{j}\right| \Psi_{j}\left(\operatorname{sgn}\left(t_{j}\right) \xi\right)\right) \quad \forall \xi \in \mathbb{R}^{d} .
$$

For every $x \in \mathbb{R}^{d}$, let $|x|:=\max _{1 \leq k \leq d}\left|x_{k}\right|$ denote the $l^{\infty}$ norm of $x$. Then $B(x, \varepsilon):=\left\{y \in \mathbb{R}^{d}:|x-y|<\varepsilon\right\}$ is the $l^{\infty}$ open ball centered at $x$ with radius $\varepsilon>0$. For every $\varepsilon>0$, define

$$
\kappa_{\varepsilon}(t):=\mathbb{P}\{\tilde{X}(t) \in B(0, \varepsilon)\} .
$$

It follows that $\kappa_{\varepsilon}(t)$ is nondecreasing in $\varepsilon$ for every fixed $t$. Moreover, the standard theory of Lévy processes ensures that $\left\{X_{j}\left(t_{j}\right)\right\}_{t_{j} \geq 0}$ is continuous in probability for all $1 \leq j \leq N$. Thus $\kappa_{\varepsilon}(t)$ is continuous in $t$ for every fixed $\varepsilon$. We consider the family of functions $\kappa:=\left\{\kappa_{\varepsilon}\right\}_{\varepsilon>0}$.

Definition 2.1. For every bounded Borel set $F \subseteq \mathbb{R}^{N}$, the box dimension profile $\overline{\operatorname{dim}}_{\kappa} F$ of $F$ with respect to the family $\kappa$ is defined as

$$
\overline{\operatorname{dim}}_{\kappa} F:=\sup \left\{\eta>0: \underline{\lim }_{\varepsilon \downarrow 0} \varepsilon^{-\eta} \inf _{\mu \in \mathcal{P}(F)} \iint \kappa_{\varepsilon}(t-s) \mu(d s) \mu(d t)=0\right\},
$$

where $\mathcal{P}(F)$ denotes the collection of all Borel probability measures supported on $F$. 
Remark 2.2. When $N=1$, we have

$$
\kappa_{\varepsilon}(t)=\mathbb{P}\{X(|t|) \in B(0, \varepsilon)\}=\mathbb{P}\{X(t) \in B(0, \varepsilon)\},
$$

for all $\varepsilon>0$ and $t \geq 0$. This shows that our definition of box dimension profiles generalize that of Khoshnevisan, Schilling, and Xiao 9 . For this reason, throughout this paper, we will use the same notation for dimension profiles as those in 9 .

A routine check shows that $\overline{\operatorname{dim}}_{\kappa}$ has the following properties:

(i) $\overline{\operatorname{dim}}_{\kappa}$ is monotone, that is, $\overline{\operatorname{dim}}_{\kappa} F \leq \overline{\operatorname{dim}}_{\kappa} G$ if $F \subseteq G$;

(ii) $\overline{\operatorname{dim}}_{\kappa}$ is finitely stable, that is, $\overline{\operatorname{dim}}_{\kappa} \bigcup_{l=1}^{n} G_{l}=\max _{1 \leq l \leq n} \overline{\operatorname{dim}}_{\kappa} G_{l}$;

(iii) $\overline{\operatorname{dim}}_{\kappa}$ is translation invariant, that is, $\overline{\operatorname{dim}}_{\kappa} F=\overline{\operatorname{dim}}_{\kappa}(F+t)$ for all $t \in \mathbb{R}^{N}$, where $=\{t+s: s \in F\}$.

We may also express $\overline{\operatorname{dim}}_{\kappa} F$ in potential-theoretic terms:

$$
\overline{\operatorname{dim}}_{\kappa} F=\varlimsup_{\varepsilon \downarrow 0} \frac{\log Z_{\kappa}(\varepsilon ; F)}{\log \varepsilon},
$$

where $Z_{\kappa}(\varepsilon ; F)$ is the minimum $\kappa_{\varepsilon}$-energy over $F$ :

$$
Z_{\kappa}(\varepsilon ; F):=\inf _{\mu \in \mathcal{P}(F)} \iint \kappa_{\varepsilon}(t-s) \mu(d s) \mu(d t) .
$$

The following technical lemma simplifies the calculation of $Z_{\kappa}(\varepsilon ; F)$. It is similar to Theorem 4.1 of Khoshnevisan and Xiao [13.

Lemma 2.3. For every bounded Borel set $F \subseteq \mathbb{R}^{N}$, let $\bar{F}$ denote the closure of $F$. Then

$$
\inf _{\mu \in \mathcal{P}(\bar{F})} \iint \kappa_{\varepsilon}(t-s) \mu(d s) \mu(d t)=\inf _{\mu \in \mathcal{P}_{f}(F)} \iint \kappa_{\varepsilon}(t-s) \mu(d s) \mu(d t),
$$

where $\mathcal{P}_{f}(F)$ denotes the collection of all finitely-supported [discrete] probability measures on $F$.

Proof. It suffices to prove

$$
\inf _{\nu \in \mathcal{P}_{f}(F)} \iint \kappa_{\varepsilon}(t-s) \nu(d s) \nu(d t) \leq \inf _{\mu \in \mathcal{P}(\bar{F})} \iint \kappa_{\varepsilon}(t-s) \mu(d s) \mu(d t),
$$

as the converse inequality is trivial. Choose and fix any $\mu \in \mathcal{P}(\bar{F})$. For every $n \geq 1$, define

$$
I_{n}(\bar{k}):=\left[\frac{k^{1}}{2^{n}}, \frac{k^{1}+1}{2^{n}}\right) \times \cdots \times\left[\frac{k^{N}}{2^{n}}, \frac{k^{N}+1}{2^{n}}\right),
$$

where $\bar{k}=\left(k^{1}, \ldots, k^{N}\right) \in \mathbb{Z}^{N}$. Let

$$
K_{n}(F):=\left\{\bar{k} \in \mathbb{Z}^{N}: I_{n}(\bar{k}) \cap \bar{F} \neq \emptyset\right\} .
$$

Note that $K_{n}(F)$ is finite since $F$ is bounded. For each $\bar{k} \in K_{n}(F)$, choose some $t_{n, \bar{k}} \in I_{n}(\bar{k}) \cap \bar{F}$. Define a new probability measure $\mu_{n}$ supported on $\left\{t_{n, \bar{k}}: \bar{k} \in\right.$ $\left.K_{n}(F)\right\}$ by

$$
\mu_{n}\left(t_{n, \bar{k}}\right)=\mu\left(I_{n}(\bar{k}) \cap \bar{F}\right) .
$$


In other words, we concentrate the mass $\mu\left(I_{n}(\bar{k}) \cap \bar{F}\right)$ to the singleton $t_{n, \bar{k}}$. Since $\kappa_{\varepsilon}(t)$ is continuous in $t$ for fixed $\varepsilon>0$ and $\bar{F}$ is compact, $\kappa_{\varepsilon}(t)$ is uniformly continuous on $\bar{F}$. Therefore

$$
\lim _{n \rightarrow \infty}\left|\iint \kappa_{\varepsilon}(t-s) \mu(d s) \mu(d t)-\iint \kappa_{\varepsilon}(t-s) \mu_{n}(d s) \mu_{n}(d t)\right|=0 .
$$

Next, since $F$ is dense in $\bar{F}$, for each $t_{n, \bar{k}}$ we can choose some $s_{n, \bar{k}} \in F$ such that $\left|t_{n, \bar{k}}-s_{n, \bar{k}}\right|<2^{-(n+1)}$. To avoid complication, we can require the $s_{n, \bar{k}}$ 's to be distinct. Define a new probability measure $\nu_{n}$ on $\left\{s_{n, \bar{k}}: \bar{k} \in K_{n}(F)\right\}$ by

$$
\nu_{n}\left(s_{n, \bar{k}}\right)=\mu_{n}\left(t_{n, \bar{k}}\right) .
$$

It follows immediately that $\nu_{n} \in \mathcal{P}_{f}(F)$. By the uniform continuity of $\kappa_{\varepsilon}(t)$ and (2.14), we have

$$
\lim _{n \rightarrow \infty}\left|\iint \kappa_{\varepsilon}(t-s) \nu_{n}(d s) \nu_{n}(d t)-\iint \kappa_{\varepsilon}(t-s) \mu(d s) \mu(d t)\right|=0 .
$$

This further implies that

$$
\inf _{\nu \in \mathcal{P}_{f}(F)} \iint \kappa_{\varepsilon}(t-s) \nu(d s) \nu(d t) \leq \iint \kappa_{\varepsilon}(t-s) \mu(d s) \mu(d t) .
$$

Since $\mu \in \mathcal{P}(\bar{F})$ is arbitrary, we can take inf over $\mathcal{P}(\bar{F})$ on the right hand side of the above inequality to complete the proof.

Lemma 2.4. For every bounded Borel set $F \subseteq \mathbb{R}^{N}$, let $\bar{F}$ denote the closure of $F$. Then

$$
\overline{\operatorname{dim}}_{\kappa} F=\overline{\operatorname{dim}}_{\kappa} \bar{F} .
$$

Proof. Since $\mathcal{P}_{f}(F) \subseteq \mathcal{P}(F) \subseteq \mathcal{P}(\bar{F})$, Lemma 2.3 implies that

$$
\inf _{\mu \in \mathcal{P}(\bar{F})} \iint \kappa_{\varepsilon}(t-s) \mu(d s) \mu(d t)=\inf _{\mu \in \mathcal{P}(F)} \iint \kappa_{\varepsilon}(t-s) \mu(d s) \mu(d t) .
$$

Then (2.18) follows from Definition 2.1

Remark 2.5. The above lemma implies that $\overline{\operatorname{dim}}_{\kappa}$ is not $\sigma$-stable in general. For example, consider $F=\mathbb{Q}_{+}^{N} \cap[0,1]^{N}$. On one hand, for every $t \in \mathbb{R}^{N}$ the only Borel probability measure supported on $\{t\}$ is the Dirac measure $\delta_{t}$. Then we can compute $\overline{\operatorname{dim}}_{\kappa}\{t\}$ by definition and obtain $\overline{\operatorname{dim}}_{\kappa}\{t\}=0$. On the other hand, $\overline{\operatorname{dim}}_{\kappa} F=\overline{\operatorname{dim}}_{\kappa} \bar{F}=\overline{\operatorname{dim}}_{\kappa}[0,1]^{N}$ according to Lemma 2.4. We will show in Corollary 4.1 that if $\kappa$ is determined by a $d$-dimensional isotropic additive $\alpha$-stable process $X$ with $0<\alpha \leq 2$, then $\overline{\operatorname{dim}}_{\kappa}[0,1]^{N}=\alpha \operatorname{Dim}_{d / \alpha}^{\mathrm{FH}}[0,1]^{N}$, where $\operatorname{Dim}_{s}^{\mathrm{FH}}$ denotes the $s$-dimensional packing dimension profile of Falconer and Howroyd [4]. Since $\operatorname{Dim}_{d / \alpha}^{\mathrm{FH}}[0,1]^{N}=\min (d / \alpha, N)>0$, we see that $\overline{\operatorname{dim}}_{\kappa} F>\sup _{t \in F} \overline{\operatorname{dim}}_{\kappa}\{t\}$.

2.2. An equivalent definition of box dimension profiles. In general it is hard to use Definition 2.1 to compute the box dimension profile of any given set since the hitting probability $\mathbb{P}\{\tilde{X}(t) \in B(0, \varepsilon)\}$ is only explicitly computable when $X$ has nice properties. However, we may express a box dimension profile in terms of the characteristic exponent $\Psi$ of the underlying additive Lévy process $X$. 
For every Borel probability measure $\mu$ on $\mathbb{R}^{N}$, and for all $\xi \in \mathbb{R}^{d}$, define the "energy form":

$$
\mathcal{E}_{\mu}(\xi):=\iint_{\mathbb{R}_{+}^{N} \times \mathbb{R}_{+}^{N}} \exp \left(-\sum_{j=1}^{N}\left|t_{j}-s_{j}\right| \Psi_{j}\left(\operatorname{sgn}\left(t_{j}-s_{j}\right) \xi\right)\right) \mu(d s) \mu(d t) .
$$

An application of Fubini's theorem shows that

$$
\begin{aligned}
\mathcal{E}_{\mu}(\xi) & =\iint_{\mathbb{R}_{+}^{N} \times \mathbb{R}_{+}^{N}} \mathbb{E}\left[e^{i \xi \cdot(X(t)-X(s))}\right] \mu(d s) \mu(d t) \\
& =\mathbb{E}\left[\left|\int e^{i \xi \cdot X(t)} \mu(d t)\right|^{2}\right] .
\end{aligned}
$$

Thus it follows that $0 \leq \mathcal{E}_{\mu}(\xi) \leq 1$.

The following proposition uses the characteristic exponent of $X$ to compute a box dimension profile. It is a generalization of Theorem 2.6 of $[9$ to the multiparameter setting. The proof of Theorem 2.6 in [9] works here and needs minor changes. See also the proof of Theorem 1.1 in Khoshnevisan and Xiao [12.

Proposition 2.6. For every compact set $F \subseteq \mathbb{R}^{N}$,

$$
\overline{\operatorname{dim}}_{\kappa} F=\sup \left\{\eta>0: \frac{\lim }{\varepsilon \downarrow 0} \varepsilon^{-\eta} \inf _{\mu \in \mathcal{P}(F)} \int_{\mathbb{R}^{d}} \frac{\mathcal{E}_{\mu}(\xi / \varepsilon)}{\prod_{k=1}^{d}\left(1+\xi_{k}^{2}\right)} d \xi=0\right\} .
$$

2.3. Packing dimension profiles. We can regularize the box dimension profiles $\overline{\operatorname{dim}}_{\kappa}$ defined in (2.5) to produce a family of packing type dimension profiles.

Definition 2.7. For every Borel set $F \subseteq \mathbb{R}^{N}$, the packing dimension profile $\operatorname{Dim}_{\kappa} F$ of $F$ with respect to the family $\kappa$ is defined as

$$
\operatorname{Dim}_{\kappa} F:=\inf \left\{\sup _{k \geq 1} \overline{\operatorname{dim}}_{\kappa} F_{k}: F \subseteq \bigcup_{k \geq 1} F_{k}, F_{k} \text { is a bounded Borel set }\right\} .
$$

One can verify that $\operatorname{Dim}_{\kappa}$ has the following properties:

(i) $\operatorname{Dim}_{\kappa}$ is monotone, that is, $\operatorname{Dim}_{\kappa} F \leq \operatorname{Dim}_{\kappa} G$ if $F \subseteq G$;

(ii) $\operatorname{Dim}_{\kappa}$ is $\sigma$-stable, that is, $\operatorname{Dim}_{\kappa} \bigcup_{n=1}^{\infty} G_{n}=\sup _{n \geq 1} \operatorname{Dim}_{\kappa} G_{n}$.

We can also associate $\operatorname{Dim}_{\kappa}$ to a "packing measure with respect to the family $\kappa "$. In order to do that, we borrow some ideas from Howroyd [6].

Definition 2.8. For every Borel set $F \subseteq \mathbb{R}^{N}$ and real number $\delta>0$, we say that a sequence of triples $\left(\omega_{i}, t_{i}, \varepsilon_{i}\right)_{i \geq 1}$ is a $(\kappa, \delta)$-packing of $F$ if for all $i \geq 1$ : (i) $\omega_{i} \geq 0$; (ii) $t_{i} \in F$; (iii) $0<\varepsilon_{i} \leq \delta$; and (iv) $\sum_{j \geq 1} \omega_{j} \kappa_{\varepsilon_{j}}\left(t_{i}-t_{j}\right) \leq 1$.

Definition 2.9. For every constant $s>0$, the $s$-dimensional packing measure $P^{s, \kappa}(F)$ of $F \subseteq \mathbb{R}^{N}$ with respect to the family $\kappa$ is defined as

$$
P^{s, \kappa}(F):=\inf \left\{\sum_{k \geq 1} P_{0}^{s, \kappa}\left(F_{k}\right): F \subseteq \bigcup_{k \geq 1} F_{k}, F_{k} \text { is a bounded Borel set }\right\}
$$

where $P_{0}^{s, \kappa}$ is a premeasure defined by

$$
P_{0}^{s, \kappa}(F):=\lim _{\delta \downarrow 0} P_{\delta}^{s, \kappa}(F)
$$


and

$$
P_{\delta}^{s, \kappa}(F):=\sup \left\{\sum_{i \geq 1} \omega_{i} \varepsilon_{i}^{s}:\left(\omega_{i}, t_{i}, \varepsilon_{i}\right)_{i \geq 1} \text { is a }(\kappa, \delta) \text {-packing of } F\right\},
$$

with the convention that $\inf \emptyset:=\infty$ and $\sup \emptyset:=0$.

Definition 2.10. For every Borel set $F \subseteq \mathbb{R}^{N}$, the packing dimension profile $\mathrm{P}-\operatorname{dim}_{\kappa} F$ of $F$ with respect to the family $\kappa$ is defined as

$$
\mathrm{P}-\operatorname{dim}_{\kappa} F:=\inf \left\{s>0: P^{s, \kappa}(F)=0\right\} .
$$

The two packing dimension profiles defined in (2.23) and (2.27) coincide. This can be shown by adapting the proof of Theorem 26 of Howroyd 6 . For completeness we record it here.

First we introduce an equivalent formulation of $Z_{\kappa}(\varepsilon ; F)$ (see (2.8) ) for fixed $\varepsilon>0$ and bounded Borel set $F \subseteq \mathbb{R}^{N}$. A sequence of pairs $\left(\omega_{i}, t_{i}\right)_{i=1}^{k}$ is a size $\varepsilon$ weighted $\kappa$-packing of $F$ if for all $i=1, \ldots, k$ : (i) $t_{i} \in F$; (ii) $\omega_{i} \geq 0$; and (iii) $\sum_{j=1}^{k} \omega_{j} \kappa_{\varepsilon}\left(t_{j}-t_{i}\right) \leq 1$. Define the quantity $N_{\kappa}(\varepsilon ; F)$ by

$$
N_{\kappa}(\varepsilon ; F):=\sup \left\{\sum_{i=1}^{k} \omega_{i}:\left(\omega_{i}, t_{i}\right)_{i=1}^{k} \text { is a size } \varepsilon \text { weighted } \kappa \text {-packing of } F\right\},
$$

with the convention that $\sup \emptyset:=0$. Then the following technical lemma holds.

Lemma 2.11. For every bounded Borel set $F \subseteq \mathbb{R}^{N}$ and real number $\varepsilon>0$,

$$
N_{\kappa}(\varepsilon ; F)=1 / Z_{\kappa}(\varepsilon ; F) \text {. }
$$

Proof. On one hand, for all $\eta<N_{\kappa}(\varepsilon ; F)$, (2.28) implies the existence of a size $\varepsilon$ weighted $\kappa$-packing $\left(\omega_{i}, t_{i}\right)_{i=1}^{k}$ of $F$ such that $\sum_{i=1}^{k} \omega_{i}>\eta$. Let $W=\sum_{i=1}^{k} \omega_{i}$ and define a discrete probability measure $\mu$ on $\left\{t_{i}\right\}_{i=1}^{k}$ by $\mu\left(t_{i}\right)=\omega_{i} / W$. It follows immediately that $\mu \in \mathcal{P}(F)$. Then elementary calculation shows that

$$
Z_{\kappa}(\varepsilon ; F) \leq \sum_{i=1}^{k} \frac{\omega_{i}}{W} \sum_{j=1}^{k} \frac{\omega_{j}}{W} \kappa_{\varepsilon}\left(t_{i}-t_{j}\right) \leq \sup _{1 \leq i \leq k} \sum_{j=1}^{k} \frac{\omega_{j}}{W} \kappa_{\varepsilon}\left(t_{i}-t_{j}\right) \leq \frac{1}{W} .
$$

Therefore $N_{\kappa}(\varepsilon ; F) \leq 1 / Z_{\kappa}(\varepsilon ; F)$.

On the other hand, for all $\eta>Z_{\kappa}(\varepsilon ; F)$, Lemma 2.3 implies the existence of a sequence $\left\{t_{i}\right\}_{i=1}^{k} \subseteq F$ together with a discrete probability measure $\mu$ on $\left\{t_{i}\right\}_{i=1}^{k}$ such that $\sum_{i, j=1}^{k} \kappa_{\varepsilon}\left(t_{i}-t_{j}\right) \omega_{i} \omega_{j}<\eta$, where $\omega_{i}=\mu\left(t_{i}\right)$ for $1 \leq i \leq k$. Let $a_{i, j}=\kappa_{\varepsilon}\left(t_{i}-t_{j}\right)$. Then the $k \times k$ matrix $A:=\left(a_{i, j}\right)_{1 \leq i, j \leq k}$ is symmetric. Let $\mathbf{u}:=\left(u_{1}, \ldots, u_{k}\right)^{\prime} \in \mathbb{R}^{k}$ be a column vector and define

$$
J:=\min \left\{\mathbf{u}^{\prime} A \mathbf{u}: \mathbf{u}=\left(u_{1}, \ldots, u_{k}\right)^{\prime} \text { such that } \sum_{i=1}^{k} u_{i}=1 \text { and } u_{i} \geq 0\right\} .
$$

A fact of linear algebra (see Proposition 3 of [6] for instance) shows that if $\mathbf{u}^{*} \in \mathbb{R}_{+}^{k}$, $\sum_{i=1}^{k} u_{i}^{*}=1$ and $\mathbf{u}^{* \prime} A \mathbf{u}^{*}=J$, then $\left(A \mathbf{u}^{*}\right)_{i}=J$, provided that $u_{i}^{*}>0$. If we select the sequence of pairs $\left(v_{j}, s_{j}\right)_{j=1}^{l}$ by removing from the sequence $\left(u_{i}^{*}, t_{i}\right)_{i=1}^{k}$ all those 
pairs $\left(u_{i}^{*}, t_{i}\right)$ with $u_{i}^{*}=0$, then we see that $\left(v_{j} / J, s_{j}\right)_{j=1}^{l}$ is a size $\varepsilon$ weighted $\kappa$ packing of $F$. It follows that

$$
N_{\kappa}(\varepsilon ; F) \geq \sum_{j=1}^{l} \frac{v_{j}}{J}=\frac{1}{J} .
$$

Since $J \leq \sum_{i, j=1}^{k} \kappa_{\varepsilon}\left(t_{i}-t_{j}\right) \omega_{i} \omega_{j}<\eta$, we have $N_{\kappa}(\varepsilon ; F) \geq 1 / Z_{\kappa}(\varepsilon ; F)$. This completes the proof.

Proposition 2.12. For every Borel set $F \subseteq \mathbb{R}^{N}$, we have

$$
\operatorname{Dim}_{\kappa} F=\mathrm{P}-\operatorname{dim}_{\kappa} F \text {. }
$$

Proof. Consider an arbitrary bounded Borel set $E \subseteq \mathbb{R}^{N}$. For each $\varepsilon>0$, let $\left(\omega_{i}, t_{i}\right)_{i=1}^{k}$ be a size $\varepsilon$ weighted $\kappa$-packing of $E$. Then $\left(\omega_{i}, t_{i}, \varepsilon\right)_{i=1}^{k}$ is trivially a $(\kappa, \varepsilon)$-packing of $E$. It follows from this fact, (2.26), and (2.28) that for all $s>0$,

$$
P_{\varepsilon}^{s, \kappa}(E) \geq \varepsilon^{s} N_{\kappa}(\varepsilon ; E)=\varepsilon^{s}\left(Z_{\kappa}(\varepsilon ; E)\right)^{-1},
$$

where we used Lemma 2.11 to derive the last equality. Now if $0<\eta<\operatorname{Dim}_{\kappa} F$, then for every sequence of bounded Borel sets $\left\{F_{k}\right\}_{k \geq 1}$ with $F \subseteq \bigcup_{k>1} F_{k}$, we can find some $F_{n}$ such that $\overline{\operatorname{dim}}_{\kappa} F_{n}>\eta$. Combining with (2.34) and (2.7), we get $P_{0}^{\eta, \kappa}\left(F_{n}\right)>0$. Therefore P-dim ${ }_{\kappa} F \geq \eta$. Let $\eta \uparrow \operatorname{Dim}_{\kappa} F$ to obtain $\operatorname{Dim}_{\kappa} F \leq$ $\mathrm{P}-\operatorname{dim}_{\kappa} F$.

On the other hand, if $0<\eta<\mathrm{P}-\operatorname{dim}_{\kappa} F$, then for every sequence of bounded Borel sets $\left\{F_{k}\right\}_{k \geq 1}$ with $F \subseteq \bigcup_{k \geq 1} F_{k}$, we can find some $F_{n}$ such that $P_{0}^{\eta, \kappa}\left(F_{n}\right)>0$. For every fixed $\theta$ satisfying $0<\theta<\eta$, we can find some $a$ such that $0<a<$ $P_{0}^{\eta, \kappa}\left(F_{n}\right)$. Since $P_{\varepsilon}^{\eta, \kappa}\left(F_{n}\right)$ decreases to $P_{0}^{\eta, \kappa}\left(F_{n}\right)$ as $\varepsilon \downarrow 0$, we have $P_{\varepsilon}^{\eta, \kappa}\left(F_{n}\right)>a$ for each $\varepsilon>0$. Then (2.26) implies the existence of a $(\kappa, \varepsilon)$-packing $\left(\omega_{i}, t_{i}, r_{i}\right)_{i=1}^{\infty}$ of $F_{n}$ such that $\sum_{i=1}^{\infty} \omega_{i} r_{i}^{\eta}>a$. For each $m \geq 1$, define

$$
K_{m}=\sum_{\left\{i: 2^{-m}<r_{i} \leq 2^{1-m}\right\}} \omega_{i} .
$$

Then there must be a positive integer $M(\varepsilon)$ such that

$$
K_{M(\varepsilon)}>2^{(M(\varepsilon)-1) \theta}\left(1-2^{\theta-\eta}\right) a,
$$

since otherwise $\sum_{m=1}^{\infty} K_{m} 2^{(1-m) \eta} \leq \sum_{m=1}^{\infty} 2^{(m-1) \theta}\left(1-2^{\theta-\eta}\right) a 2^{(1-m) \eta}=a$. Let $\{i(j)\}_{j=1}^{J}$ be a subsequence such that $2^{-M(\varepsilon)}<r_{i(j)} \leq 2^{1-M(\varepsilon)}$ for all $1 \leq j \leq J$ and $\sum_{j=1}^{J} \omega_{i(j)} \geq 2^{(M(\varepsilon)-1) \theta}\left(1-2^{\theta-\eta}\right) a$. The fact that $\left(\omega_{i}, t_{i}, r_{i}\right)_{i=1}^{\infty}$ is a $(\kappa, \varepsilon)$ packing of $F_{n}$ implies that $\sum_{j \geq 1} \omega_{j} \kappa_{r_{j}}\left(t_{i}-t_{j}\right) \leq 1$ for all $i \geq 1$. Since $\kappa_{\varepsilon}(t)$ is nondecreasing in $\varepsilon$ for fixed $t$ and $r_{i(j)} \leq 2^{1-M(\varepsilon)}$ for all $1 \leq j \leq J$, we see that $\left(\omega_{i(j)}, t_{i(j)}\right)_{j=1}^{J}$ is a size $2^{-M(\varepsilon)}$ weighted $\kappa$-packing of $F_{n}$. Therefore

$$
N_{\kappa}\left(2^{-M(\varepsilon)} ; F_{n}\right) \geq 2^{(M(\varepsilon)-1) \theta}\left(1-2^{\theta-\eta}\right) a .
$$

Since $M(\varepsilon) \uparrow \infty$ as $\varepsilon \downarrow 0$, by taking logarithms and then limsup as $\varepsilon \downarrow 0$, we obtain

$$
\overline{\operatorname{dim}}_{\kappa} F_{n} \geq \varlimsup_{\varepsilon \downarrow 0} \frac{\log Z_{\kappa}\left(2^{-M(\varepsilon)} ; F_{n}\right)}{\log 2^{-M(\varepsilon)}}=\varlimsup_{M \rightarrow \infty} \frac{\log N_{\kappa}\left(2^{-M} ; F_{n}\right)}{-\log 2^{-M}} \geq \theta .
$$

Let $\theta \uparrow \eta$ to see that $\operatorname{Dim}_{\kappa} F \geq \mathrm{P}-\operatorname{dim}_{\kappa} F$. This completes the proof. 
The connection between packing dimension profiles $\operatorname{Dim}_{\kappa}$ and the family of measures $\left\{P^{s, \kappa}\right\}_{s \geq 0}$ can be used to prove the following technical lemma, which will be used later.

Lemma 2.13. If a Borel set $F \subseteq \mathbb{R}^{N}$ satisfies $\operatorname{Dim}_{\kappa} F>s$ for some $s \geq 0$, then there exists a compact set $K \subseteq F$ such that $\operatorname{Dim}_{\kappa}(K \cap G) \geq s$ for all open sets $G \subseteq \mathbb{R}^{N}$ with $K \cap G \neq \emptyset$.

Proof. Since $\operatorname{Dim}_{\kappa} F>s$, for every collection of bounded Borel sets $\left\{F_{n}\right\}_{n \geq 1}$ with $F \subseteq \bigcup_{n>1} F_{n}$, we can find some $F_{n}$ such that $\operatorname{Dim}_{\kappa} F_{n}>s$. This implies $P^{s, \kappa}\left(F_{n}\right)=$ $\infty$. Then we can use the proof of Theorem 22 of Howroyd [6] to complete the argument.

2.4. Main result. With the box dimension profiles and packing dimension profiles defined so far, we are able to present our main result regarding the packing dimension of images of additive Lévy processes.

Theorem 2.14. Let $X:=\left\{X(t): t \in \mathbb{R}_{+}^{N}\right\}$ be an additive Lévy process in $\mathbb{R}^{d}$ and let $\overline{\operatorname{dim}}_{\mathrm{M}}$ denote the upper box-counting dimension. Then for all nonrandom bounded Borel sets $F \subseteq \mathbb{R}_{+}^{N}$ :

$$
\begin{aligned}
& \overline{\operatorname{dim}}_{\mathrm{M}} X(F)=\overline{\operatorname{dim}}_{\kappa} F \text { a.s. } \text { and } \\
& \operatorname{dim}_{\mathrm{P}} X(F)=\operatorname{Dim}_{\kappa} F \text { a.s. }
\end{aligned}
$$

We will prove this theorem in the next section.

\section{Proof OF THE MAIN THEOREM}

In this section we strive to prove Theorem 2.14 The proof relies on finding upper bounds for the probabilities of the events where the underlying additive Lévy process hits small balls. In order to estimate these probabilities, we adapt the methods of section 4 of Khoshnevisan, Xiao, and Zhong [14. We first borrow certain results from [14] without proofs.

3.1. Preliminaries. Recall that $X=\left\{X_{t}: t \in \mathbb{R}_{+}^{N}\right\}$ is an additive Lévy process on $\mathbb{R}^{d}$. We assume that the underlying probability space is $(\Omega, \mathcal{F}, \mathbb{P})$. Define $\mathbb{P}_{x}$ to be the law of $x+X$ for any $x \in \mathbb{R}^{d}$, and let $\mathbb{E}_{x}$ be the corresponding expectation operator. Furthermore, we define a sigma-finite measure $\mathbb{P}_{\lambda_{d}}$ and a corresponding expectation operator $\mathbb{E}_{\lambda_{d}}$ by

$$
\begin{array}{ll}
\mathbb{P}_{\lambda_{d}}\{A\}=\int_{\mathbb{R}^{d}} \mathbb{P}_{x}\{A\} d x & \forall A \subseteq \mathbb{R}^{d} \text { Borel, } \\
\mathbb{E}_{\lambda_{d}}[Z]=\int_{\mathbb{R}^{d}} \mathbb{E}_{x}[Z] d x & \forall Z: \Omega \rightarrow \mathbb{R}_{+} \text {bounded measurable. }
\end{array}
$$

We also extend the idea of filtrations. Let $\Pi=\{1, \ldots, N\}$. For all $A \subseteq \Pi$ define the partial order $\preccurlyeq(A)$ on $\mathbb{R}^{N}$ by

$$
s \preccurlyeq(A) t \Longleftrightarrow \begin{cases}s_{i} \leq t_{i}, & \text { for all } i \in A, \\ s_{i}>t_{i}, & \text { for all } i \in A^{C} .\end{cases}
$$

We may also write $t \succcurlyeq_{(A)} s$ for $s \preccurlyeq(A) t$. It follows that the partial orders $\{\preccurlyeq(A)$ : $A \subseteq \Pi\}$ totally order $\mathbb{R}^{N}$ in the sense that, for all $s, t \in \mathbb{R}^{N}$, we can find $A \subseteq \Pi$ such that $s \preccurlyeq(A) t$. For each $A \subseteq \Pi$, we define $\mathcal{F}^{A}(t)$ to be the $\sigma$-field generated 
by $\{X(r): r \preccurlyeq(A) t\}$. We can, and will assume, that $\mathcal{F}^{A}(t)$ is $\mathbb{P}_{x^{-}}$-complete for all $x \in \mathbb{R}^{d}$, and each $\mathcal{F}^{A}(t)$ is $\preccurlyeq(A)$-right continuous.

For any Borel probability measure $\mu \in \mathcal{P}\left(\mathbb{R}_{+}^{N}\right)$, define the occupation measure $\mathbb{O}_{\mu}$ on $\mathbb{R}^{d}$ via the prescription

$$
\mathbb{O}_{\mu}(A):=\int_{\mathbb{R}_{+}^{N}} \mathbb{1}_{A}(X(s)) \mu(d s) \quad \forall \text { Borel set } A \subseteq \mathbb{R}^{d} .
$$

Then by standard integration theory, we have

$$
\mathbb{O}_{\mu}(f)=\int_{\mathbb{R}_{+}^{N}} f(X(s)) \mu(d s),
$$

for all nonnegative measurable or bounded measurable function $f: \mathbb{R}^{d} \rightarrow \mathbb{R}$. Now for fixed $\mu \in \mathcal{P}\left(\mathbb{R}_{+}^{N}\right)$, bounded or nonnegative function $f: \mathbb{R}^{d} \rightarrow \mathbb{R}$, and $A \subseteq \Pi$, define the process $\mathcal{M}_{\mu}^{A} f$ by

$$
\mathcal{M}_{\mu}^{A} f(t):=\mathbb{E}_{\lambda_{d}}\left[\mathbb{O}_{\mu}(f) \mid \mathcal{F}^{A}(t)\right] \quad \forall t \in \mathbb{R}_{+}^{N} .
$$

The following lemma is from Lemma 4.2 of [14].

Lemma 3.1. For all measurable functions $f: \mathbb{R}^{d} \rightarrow \mathbb{R}_{+}$in $L^{1}\left(\mathbb{R}^{d}\right) \cap L^{2}\left(\mathbb{R}^{d}\right)$ with its Fourier transform $\hat{f} \in L^{1}\left(\mathbb{R}^{d}\right)$, and for all $\mu \in \mathcal{P}\left(\mathbb{R}_{+}^{N}\right)$, the following holds:

$$
\mathbb{E}_{\lambda_{d}}\left[\sup _{s \in \mathbb{Q}_{+}^{N}}\left|\mathcal{M}_{\mu}^{A} f(s)\right|^{2}\right] \leq 4^{N}(2 \pi)^{-d} \int_{\mathbb{R}^{d}}|\hat{f}(\xi)|^{2} \mathcal{E}_{\mu}(\xi) d \xi,
$$

where $\mathcal{E}_{\mu}$ is defined in (2.20).

Finally, recall the associated process $\widetilde{X}=\left\{\widetilde{X}(t): t \in \mathbb{R}_{+}^{N}\right\}$ of $X$ defined in (2.1). We define a family of operators $P=\left\{P_{t}: t \in \mathbb{R}^{N}\right\}$ on $L^{\infty}\left(\mathbb{R}^{d}\right)$ by

$$
P_{t} f(x):=\mathbb{E}[f(\tilde{X}(t)+x)] \quad \forall f \in L^{\infty}\left(\mathbb{R}^{d}\right) \text { and } x \in \mathbb{R}^{d} .
$$

Proposition 3.2 of [14] states the Markov property of $X$ in terms of $P_{t}$ and we record it here for future reference.

Lemma 3.2. Suppose that $A \subseteq \Pi$ and that $s \preccurlyeq_{(A)} t$, where $s$ and $t$ are both in $\mathbb{R}_{+}^{N}$. Then for every measurable function $f: \mathbb{R}^{d} \rightarrow \mathbb{R}_{+}$,

$$
\mathbb{E}_{\lambda_{d}}\left[f(X(t)) \mid \mathcal{F}^{A}(s)\right]=P_{t-s} f(X(s)),
$$

$\mathbb{P}_{\lambda_{d}}$-a.s., where $P_{t}$ is defined in (3.8).

3.2. Hitting probabilities. For every $\delta>0$ and Borel set $F \subseteq \mathbb{R}_{+}^{N}$, let $F^{\delta}$ denote the closed $\delta$-enlargement of $F$, that is, $F^{\delta}:=\left\{y \in \mathbb{R}_{+}^{N}: \operatorname{dist}(y, F) \leq \delta\right\}$, where $\operatorname{dist}(y, F):=\inf _{x \in F}|x-y|$. Let $\lambda_{d}$ denote the Lebesgue measure on $\mathbb{R}^{d}$. For fixed $\varepsilon>0$ and Borel set $F \subseteq \mathbb{R}_{+}^{N}$, we want to estimate $\mathbb{E}\left[\lambda_{d}\left((X(F))^{\varepsilon}\right)\right]$. It turns out that this quantity equals the probability of the event that the underlying additive Lévy process hits a small ball.

Proposition 3.3. For every bounded Borel set $F \subseteq \mathbb{R}_{+}^{N}$ and small number $\varepsilon>0$, we have

$$
\mathbb{E}\left[\lambda_{d}\left((X(F))^{\varepsilon}\right)\right] \leq \frac{C \varepsilon^{d}}{Z_{\kappa}(\varepsilon ; F)},
$$

where $Z_{\kappa}(\varepsilon ; F)$ is defined in (2.8) and the constant $C$ depends only on $N$ and $d$. 
Proof. We use the idea of the proof for Theorem 2.1 of 14 . Choose and fix some $\Delta \notin \mathbb{R}_{+}^{N}$. For each $\delta>0$, let $T^{\delta}$ denote some measurable and $\left(\mathbb{Q}_{+}^{N} \cap F^{\delta}\right) \cup\{\Delta\}$ valued function on $\Omega$, such that $T^{\delta} \neq \Delta$ if and only if $\left|X\left(T^{\delta}\right)\right|<\varepsilon / 2$. Then for each $k>0$ define a set function

$$
\mu^{\delta, k}(\cdot):=\frac{\mathbb{P}_{\lambda_{d}}\left\{T^{\delta} \in \cdot, T^{\delta} \neq \Delta,|X(0)| \leq k\right\}}{\mathbb{P}_{\lambda_{d}}\left\{T^{\delta} \neq \Delta,|X(0)| \leq k\right\}} .
$$

Since $B(0, \varepsilon)$ is open and $X_{j}$ is right-continuous for all $1 \leq j \leq N$, an application of Fubini's theorem implies that

$$
\begin{aligned}
\mathbb{P}_{\lambda_{d}}\left\{T^{\delta} \neq \Delta,|X(0)| \leq k\right\} & =\mathbb{P}_{\lambda_{d}}\left\{X\left(F^{\delta}\right) \cap B(0, \varepsilon / 2) \neq \emptyset,|X(0)| \leq k\right\} \\
& =\int_{[-k, k]^{d}} \mathbb{P}\left\{X\left(F^{\delta}\right) \cap B(-x, \varepsilon / 2) \neq \emptyset\right\} d x \\
& =\mathbb{E}\left[\lambda_{d}\left(\left(X\left(F^{\delta}\right) \oplus B(0, \varepsilon / 2)\right) \cap[-k, k]^{d}\right)\right] .
\end{aligned}
$$

Since the last term is positive and finite, we see that $\mu^{\delta, k}$ is a probability measure on $F^{\delta}$.

Now for every $A \subseteq \Pi$ and nonnegative measurable or bounded measurable function $f: \mathbb{R}^{d} \rightarrow \mathbb{R}$, we have

$$
\begin{aligned}
\mathcal{M}_{\mu^{\delta, k}}^{A} f(s) & \geq \mathbb{E}_{\lambda_{d}}\left[\int_{t \succcurlyeq(A) s} f(X(t)) \mu^{\delta, k}(d t) \mid \mathcal{F}^{A}(s)\right] \\
& =\int_{t \succcurlyeq(A)} \mathbb{E}_{\lambda_{d}}\left[f(X(t)) \mid \mathcal{F}^{A}(s)\right] \mu^{\delta, k}(d t) \\
& =\int_{t \succcurlyeq(A)} P_{t-s} f(X(s)) \mu^{\delta, k}(d t),
\end{aligned}
$$

$\mathbb{P}_{\lambda_{d}}$-almost surely, where the last equality follows from Lemma 3.2 We obtain the following by taking sup over all positive rational numbers and noticing that $T^{\delta} \in \mathbb{Q}_{+}^{N} \cup\{\Delta\}$ and $\left|X\left(T^{\delta}\right)\right|<\varepsilon / 2$ on $\left\{T^{\delta} \neq \Delta,|X(0)| \leq k\right\}$ :

$$
\begin{aligned}
& \sup _{s \in \mathbb{Q}_{+}^{N}} \mathcal{M}_{\mu^{\delta, k}}^{A} f(s) \\
& \quad \geq \sup _{s \in \mathbb{Q}_{+}^{N}} \int_{t \succcurlyeq(A) s} P_{t-s} f(X(s)) \mu^{\delta, k}(d t) \\
& \geq \int_{t \succcurlyeq(A)} T^{\delta} P_{t-T^{\delta}} f\left(X\left(T^{\delta}\right)\right) \mu^{\delta, k}(d t) \cdot \mathbb{1}\left\{T^{\delta} \neq \Delta,|X(0)| \leq k\right\} \\
& \geq \int_{t \succcurlyeq(A)} \inf _{T^{\delta}} \quad \operatorname{in}^{d},|x| \leq \varepsilon / 2 \\
& P_{t-T^{\delta}} f(x) \mu^{\delta, k}(d t) \cdot \mathbb{1}\left\{T^{\delta} \neq \Delta,|X(0)| \leq k\right\}
\end{aligned}
$$

$\mathbb{P}_{\lambda_{d}}$-almost surely. Summing over all $A \subseteq \Pi$ gives

$$
\begin{aligned}
& \sum_{A \subseteq \Pi} \sup _{s \in \mathbb{Q}_{+}^{N}} \mathcal{M}_{\mu^{\delta, k}}^{A} f(s) \\
& \quad \geq \int_{\mathbb{R}_{+}^{N}} \inf _{x \in \mathbb{R}^{d},|x| \leq \varepsilon / 2} P_{t-T^{\delta}} f(x) \mu^{\delta, k}(d t) \cdot \mathbb{1}\left\{T^{\delta} \neq \Delta,|X(0)| \leq k\right\},
\end{aligned}
$$

$\mathbb{P}_{\lambda_{d}}$-almost surely. Note that the null set is independent of the choice of $\varepsilon>0$. By squaring and taking $\mathbb{P}_{\lambda_{d}}$-expectations on both sides of the above inequality, and 
noticing the special choice of $\mu^{\delta, k}$, we obtain the following:

(3.16)

$$
\begin{aligned}
& \mathbb{E}_{\lambda_{d}}\left[\left|\sum_{A \subseteq \Pi} \sup _{s \in \mathbb{Q}_{+}^{N}} \mathcal{M}_{\mu^{\delta, k}}^{A} f(s)\right|^{2}\right] \\
& \geq \int_{\mathbb{R}_{+}^{N}}\left[\int_{\mathbb{R}_{+}^{N}} \inf _{|x| \leq \varepsilon / 2} P_{t-s} f(x) \mu^{\delta, k}(d t)\right]^{2} \mu^{\delta, k}(d s) \cdot \mathbb{P}_{\lambda_{d}}\left\{T^{\delta} \neq \Delta,|X(0)| \leq k\right\} \\
& \geq\left[\int_{\mathbb{R}_{+}^{N}} \int_{\mathbb{R}_{+}^{N}} \inf _{|x| \leq \varepsilon / 2} P_{t-s} f(x) \mu^{\delta, k}(d t) \mu^{\delta, k}(d s)\right]^{2} \cdot \mathbb{P}_{\lambda_{d}}\left\{T^{\delta} \neq \Delta,|X(0)| \leq k\right\},
\end{aligned}
$$

where the last inequality follows from the Cauchy-Schwarz inequality. On one hand, the elementary inequality $\left(\sum_{k=1}^{n} a_{k}\right)^{2} \leq n \sum_{k=1}^{n} a_{k}^{2}$ shows that

$$
\begin{aligned}
\mathbb{E}_{\lambda_{d}}\left[\left|\sum_{A \subseteq \Pi} \sup _{s \in \mathbb{Q}_{+}^{N}} \mathcal{M}_{\mu^{\delta, k}}^{A} f(s)\right|^{2}\right] & \leq 2^{N} \sum_{A \subseteq \Pi} \mathbb{E}_{\lambda_{d}}\left[\left|\sup _{s \in \mathbb{Q}_{+}^{N}} \mathcal{M}_{\mu^{\delta, k}}^{A} f(s)\right|^{2}\right] \\
& \leq 16^{N}(2 \pi)^{-d} \int_{\mathbb{R}^{d}}|\hat{f}(\xi)|^{2} \mathcal{E}_{\mu^{\delta, k}}(\xi) d \xi
\end{aligned}
$$

where we used Lemma 3.1 to derive the last inequality. On the other hand, we choose our $f$ to be the Fourier transform of the $\varepsilon$-scaled Pólya distribution, that is,

$$
f_{\varepsilon}(x)=\prod_{k=1}^{d}\left(1-\frac{\left|x_{k}\right|}{2 \varepsilon}\right)^{+} \quad \forall x \in \mathbb{R}^{d},
$$

where $a^{+}:=\max (a, 0)$ for all real numbers $a$. Then the Fourier transform of $f_{\varepsilon}$ is given by

$$
\hat{f}_{\varepsilon}(\xi)=\prod_{k=1}^{d}\left(\frac{1-\cos \left(2 \varepsilon \xi_{k}\right)}{\varepsilon \xi_{k}^{2}}\right) \quad \forall \xi \in \mathbb{R}^{d} .
$$

It follows that both $f_{\varepsilon}$ and $\hat{f}_{\varepsilon}$ are continuous, nonnegative, and in $L^{1}\left(\mathbb{R}^{d}\right) \cap L^{2}\left(\mathbb{R}^{d}\right)$. In particular, combining with (2.20), we get

$$
\int_{\mathbb{R}^{d}}|\hat{f}(\xi)|^{2} \mathcal{E}_{\mu}(\xi) d \xi \leq \int_{\mathbb{R}^{d}}|\hat{f}(\xi)|^{2} d \xi
$$

for all $\mu \in \mathcal{P}\left(\mathbb{R}_{+}^{N}\right)$, and

$$
\begin{aligned}
\int_{\mathbb{R}^{d}}|\hat{f}(\xi)|^{2} d \xi & =\int_{\mathbb{R}^{d}} \prod_{k=1}^{d}\left(\frac{1-\cos \left(2 \varepsilon \xi_{k}\right)}{\varepsilon \xi_{k}^{2}}\right)^{2} d \xi=\prod_{k=1}^{d}\left(\int_{\mathbb{R}} \frac{\left(1-\cos \left(2 \varepsilon \xi_{k}\right)\right)^{2}}{\varepsilon^{2} \xi_{k}^{4}} d \xi_{k}\right) \\
& =\left(8 \varepsilon \int_{\mathbb{R}} \frac{(1-\cos y)^{2}}{y^{4}} d y\right)^{d}=\left(8 C_{0}\right)^{d} \varepsilon^{d}
\end{aligned}
$$

where $C_{0}:=\int_{\mathbb{R}} y^{-4}(1-\cos y)^{2} d y<\infty$.

From (3.8) and (3.18), we see that $P_{t-s} f_{\varepsilon}(x)$ is continuous in $(t, s, x)$. Since $\overline{B(0, \varepsilon / 2)} \times F^{\delta_{0}} \times F^{\delta_{0}}$ is compact (say $\delta_{0}=1$ ), $P_{t-s} f_{\varepsilon}(x)$ is uniformly continuous on $\overline{B(0, \varepsilon / 2)} \times F^{\delta_{0}} \times F^{\delta_{0}}$. In particular, $g(t, s):=\inf _{x \in \mathbb{R}^{d},|x| \leq \varepsilon / 2} P_{t-s} f_{\varepsilon / 2}(x)$ is 
continuous on $F^{\delta_{0}} \times F^{\delta_{0}}$. Since the family of probability measures $\left\{\mu^{1 / n, m}\right\}_{n, m \geq 1}$ are tight, and $F^{\delta} \downarrow \bar{F}$ as $\delta \downarrow 0$, an application of Prohorov's theorem, together with the continuity of $\inf _{x} P_{t-s} f_{\varepsilon / 2}(x)$, imply that there exists some $\mu \in \mathcal{P}(\bar{F})$, such that, along certain subsequence $\delta^{\prime} \downarrow 0$ and $k^{\prime} \uparrow \infty$,

$$
\begin{gathered}
\iint \inf _{|x| \leq \varepsilon / 2} P_{t-s} f_{\varepsilon}(x) \mu^{\delta^{\prime}, k^{\prime}}(d t) \mu^{\delta^{\prime}, k^{\prime}}(d s) \\
\rightarrow \iint \inf _{|x| \leq \varepsilon / 2} P_{t-s} f_{\varepsilon}(x) \mu(d t) \mu(d s) .
\end{gathered}
$$

Furthermore, (3.12) shows that

$$
\lim _{\delta \downarrow 0} \lim _{k \uparrow \infty} \mathbb{P}_{\lambda_{d}}\left\{T^{\delta} \neq \Delta,|X(0)| \leq k\right\}=\mathbb{E}\left[\lambda_{d}\left((X(\bar{F}))^{\varepsilon / 2}\right)\right] .
$$

Here $\bar{F}$ denotes the closure of $F$. Now we combine (3.16), (3.17), (3.21), (3.22) and (3.23) to get

$$
\mathbb{E}\left[\lambda_{d}\left((X(\bar{F}))^{\varepsilon / 2}\right)\right] \cdot \iint \inf _{|x| \leq \varepsilon / 2} P_{t-s} f_{\varepsilon}(x) \mu(d t) \mu(d s) \leq 16^{N}(2 \pi)^{-d}\left(8 C_{0}\right)^{d} \varepsilon^{d},
$$

where $C_{0}=\int_{\mathbb{R}} y^{-4}(1-\cos y)^{2} d y<\infty$.

Finally, we notice that $1-(2 \varepsilon)^{-1}|z| \geq \frac{1}{2}$ whenever $z \in B(0, \varepsilon)$. Consequently, $2^{-d} \mathbb{1}_{B(0, \varepsilon)}(z) \leq f_{\varepsilon}(z)$ for all $z \in \mathbb{R}^{d}$. Let $z:=\tilde{X}(t-s)+x$, use (3.8), and take expectation to find

$$
\begin{aligned}
P_{t-s} f_{\varepsilon}(x) & =\mathbb{E}\left[f_{\varepsilon}(\tilde{X}(t-s)+x)\right] \\
& \geq 2^{-d} \mathbb{E}\left[\mathbb{1}_{B(0, \varepsilon)}(\tilde{X}(t-s)+x)\right]=2^{-d} \mathbb{P}\{|\tilde{X}(t-s)+x| \leq \varepsilon\} \\
& \geq 2^{-d} \mathbb{P}\{|\tilde{X}(t-s)|<\varepsilon / 2\} \cdot \mathbb{1}\{|x| \leq \varepsilon / 2\},
\end{aligned}
$$

where the last line follows from the triangle inequality. Taking inf over $|x| \leq \varepsilon / 2$ gives

$$
\inf _{x \in \mathbb{R}^{d},|x| \leq \varepsilon / 2} P_{t-s} f_{\varepsilon}(x) \geq 2^{-d} \mathbb{P}\{|\widetilde{X}(t-s)|<\varepsilon / 2\}=2^{-d} \kappa_{\varepsilon / 2}(t-s) .
$$

We combine the above inequality with (3.24) and change $\varepsilon / 2$ to $\varepsilon$ to obtain

$$
\mathbb{E}\left[\lambda_{d}\left((X(\bar{F}))^{\varepsilon}\right)\right] \leq \frac{16^{N}(2 \pi)^{-d}\left(8 C_{0}\right)^{d} 2^{d}(2 \varepsilon)^{d}}{\inf _{\nu \in \mathcal{P}(\bar{F})} \iint \kappa_{\varepsilon}(t-s) \nu(d t) \nu(d s)} .
$$

The proposition is proved, thanks to Lemma 2.3 .

3.3. Proof of Theorem 2.14. With the help of Proposition 3.3. we can adapt the proof of Theorem 2.7 of Khoshnevisan, Schilling, and Xiao [9] for one-parameter Lévy processes to the current multiparameter setting. Equation (2.39) can be proved with minor changes and we only prove equation (2.40). For that purpose, we need the following technical lemma.

Lemma 3.4. For all compact sets $F \subseteq \mathbb{R}_{+}^{N}, \operatorname{dim}_{\mathrm{P}}(\overline{X(F)})=\operatorname{dim}_{\mathrm{P}}(X(F))$ almost surely, where $\bar{E}$ denotes the closure of a set $E$.

Proof. It is straightforward to see that $\operatorname{dim}_{\mathrm{P}}(\overline{X(F)}) \geq \operatorname{dim}_{\mathrm{P}}(X(F))$ almost surely. Then we recall that $X(t)=\sum_{j=1}^{N} X_{j}\left(t_{j}\right)$ for $t \in \mathbb{R}_{+}^{N}$ and each $X_{j}$ is a one-parameter 
Lévy process. Notice that with probability one

$$
\{t \in F: X \text { is discontinuous at } t\} \subseteq \bigcup_{j=1}^{N}\left\{t \in F: X_{j}\left(t_{j}\right) \neq X_{j}\left(t_{j}-\right)\right\} .
$$

Furthermore, for each $1 \leq j \leq N$, let $Y^{j}(t):=\sum_{1<l<N, l \neq j} X_{l}\left(t_{l}\right)+X_{j}\left(t_{j}-\right)$. Since there are at most countably many jumps for each $X_{j}$, and $\operatorname{dim}_{\mathrm{P}}$ is $\sigma$-stable, it follows that for all $1 \leq j \leq N$,

$$
\begin{aligned}
& \operatorname{dim}_{\mathrm{P}}\left(\left\{Y^{j}(t): t \in F, X_{j}\left(t_{j}\right) \neq X_{j}\left(t_{j}-\right)\right\}\right) \\
& \quad=\operatorname{dim}_{\mathrm{P}}\left(\left\{X(t): t \in F, X_{j}\left(t_{j}\right) \neq X_{j}\left(t_{j}-\right)\right\}\right) \leq \operatorname{dim}_{\mathrm{P}} X(F) \quad \text { a.s. }
\end{aligned}
$$

Since $\overline{X(F)} \subseteq X(F) \cup\left(\bigcup_{j=1}^{N}\left\{Y^{j}(t): t \in F, X_{j}\left(t_{j}\right) \neq X_{j}\left(t_{j}-\right)\right\}\right.$ almost surely, the $\sigma$-stability of $\operatorname{dim}_{\mathrm{P}}$ implies that $\operatorname{dim}_{\mathrm{P}}(\overline{X(F)}) \leq \operatorname{dim}_{\mathrm{P}}(X(F))$ almost surely. This completes the proof.

Proof of Theorem 2.14 (2.40). Definition 2.7 implies that for every $\eta>\operatorname{Dim}_{\kappa} F$ there exists a sequence of bounded Borel sets $\left\{F_{n}\right\}_{n \geq 1}$ such that

$$
F \subseteq \bigcup_{n=1}^{\infty} F_{n} \quad \text { and } \quad \sup _{n \geq 1} \overline{\operatorname{dim}}_{\kappa} F_{n}<\eta
$$

Since $X(F) \subseteq \bigcup_{n \geq 1} X\left(F_{n}\right)$, (2.39) implies that

$$
\operatorname{dim}_{\mathrm{P}} X(F) \leq \sup _{n \geq 1} \overline{\operatorname{dim}}_{\mathrm{M}} X\left(F_{n}\right)=\sup _{n \geq 1} \overline{\operatorname{dim}}_{\kappa} F_{n}<\eta \quad \text { a.s. }
$$

Let $\eta \downarrow \operatorname{Dim}_{\kappa} F$ to obtain $\operatorname{dim}_{\mathrm{P}} X(F) \leq \operatorname{Dim}_{\kappa} F$ almost surely.

Next we prove the converse inequality, that is, $\operatorname{dim}_{\mathrm{P}} X(F) \geq \operatorname{Dim}_{\kappa} F$ almost surely. Without loss of generality, we may assume that $\operatorname{Dim}_{\kappa} F>0$; otherwise, there is nothing to prove. For every $0<\eta<\operatorname{Dim}_{\kappa} F$, Lemma 2.13 implies that there exists a compact subset $K \subseteq F$, depending on $\eta$, such that

$$
\overline{\operatorname{dim}}_{\kappa}(K \cap[s, t]) \geq \eta,
$$

for all $s, t \in \mathbb{Q}_{+}^{N}$ and $s \preccurlyeq(\Pi) t$ (see (3.3)) such that $K \cap[s, t] \neq \emptyset$, where $[s, t]:=$ $\left\{r \in \mathbb{R}_{+}^{N}: s \preccurlyeq(\Pi) r \preccurlyeq(\Pi) t\right\}$. For each $1 \leq j \leq N$, let $T_{j}$ denote the set

$$
\begin{aligned}
& \left\{t \in K: X_{j}\left(t_{j}-\right) \neq X_{j}\left(t_{j}\right) \text { and there is no sequence }\left\{t^{n}\right\}_{n \geq 1} \subseteq K\right. \\
& \text { such that } \left.t_{j}^{n} \downarrow t_{j} \text { as } n \uparrow \infty\right\} .
\end{aligned}
$$

Since each $X_{j}$ has at most countably many jumps almost surely, each $T_{j}$ is the union of at most countably many hyperplanes in $\mathbb{R}^{N}$ almost surely. By a similar argument as in Lemma 3.4, we may remove the set $\bigcup_{1 \leq j \leq N}\left\{X(t): t \in T_{j}\right\}$ from $\overline{X(K)}$ such that the remaining set $\overline{X(K)} \backslash\left(\bigcup_{1 \leq j \leq N}\left\{X(t): t \in T_{j}\right\}\right)$ has the same packing dimension as $\overline{X(K)}$. Moreover $\overline{X(K)} \backslash\left(\bigcup_{1 \leq j \leq N}\left\{X(t): t \in T_{j}\right\}\right)$ is closed almost surely. Therefore, without loss of generality, we may assume that $\overline{X(K)}$ is almost surely a closed set and for every $x \in X(K)$ there is a subset $A \subseteq \Pi$ and a sequence $\left\{t^{n}\right\}_{n \geq 1} \subseteq K$ such that $t^{n} \preccurlyeq(A) t^{n+1}, t_{j}^{n} \neq t_{j}^{n+1}$ for all $1 \leq j \leq N$ and $n \geq 1$, and $x=\lim _{n \rightarrow \infty} X\left(t^{n}\right)$. Then for every fixed $a \in \mathbb{Q}^{d}$ and $r \in \mathbb{Q}_{+}$, if $\overline{X(K)} \cap B(a, r) \neq \emptyset$, then we can find (random) $s, t \in \mathbb{Q}_{+}^{N}$ such that $s \preccurlyeq(\Pi) t$, $K \cap[s, t] \neq \emptyset$, and $X(K \cap[s, t]) \subseteq B(a, r)$. Since

$$
\mathbb{P}\left\{\overline{\operatorname{dim}}_{\mathrm{M}} X(K \cap[s, t])=\overline{\operatorname{dim}}_{\kappa}(K \cap[s, t]) \text { for all } s \preccurlyeq(\Pi) t \in \mathbb{Q}_{+}^{N}\right\}=1 \text {, }
$$


from our choice of $K$ (see (3.32) ), we see that

$$
\overline{\operatorname{dim}}_{\mathrm{M}}(\overline{X(K)} \cap B(a, r)) \geq \eta \quad \text { a.s. on the event }\{\overline{X(K)} \cap B(a, r) \neq \emptyset\} .
$$

This further implies that the event

$$
\begin{gathered}
\left\{\overline{\operatorname{dim}}_{\mathrm{M}}(\overline{X(K)} \cap B(a, r)) \geq \eta \text { whenever } \overline{X(K)} \cap B(a, r) \neq \emptyset\right. \\
\text { for all } \left.a \in \mathbb{Q}^{d} \text { and } r \in \mathbb{Q}_{+}\right\}
\end{gathered}
$$

happens with probability one. Now Baire's category theorem (Proposition 3.6 of Falconer [3]) shows that $\operatorname{dim}_{\mathrm{P}} \overline{X(K)} \geq \eta$ almost surely. Finally, thanks to Lemma 3.4, we have $\operatorname{dim}_{\mathrm{P}} X(K) \geq \eta$ almost surely. Let $\eta \uparrow \operatorname{Dim}_{\kappa} F$ to obtain $\operatorname{dim}_{\mathrm{P}} X(F) \geq$ $\operatorname{Dim}_{\kappa} F$ almost surely.

\section{Applications}

In this section we apply Theorem 2.14 to certain additive Lévy processes in order to derive some interesting corollaries. First we present the promised connection between the packing dimension profiles defined in (2.23) and that of Falconer and Howroyd [4] and Howroyd [6].

Corollary 4.1. Let $X$ be a d-dimensional additive Lévy process so that each $X_{j}$ is isotropic $\alpha$-stable for some $0<\alpha \leq 2$. Then for every nonrandom bounded Borel set $F \subseteq \mathbb{R}_{+}^{N}$, we have

$$
\operatorname{Dim}_{\kappa} F=\alpha \operatorname{Dim}_{d / \alpha}^{\mathrm{FH}} F,
$$

where $\operatorname{Dim}_{s}^{\mathrm{FH}}$ denotes the s-dimension packing dimension profiles of Falconer and Howroyd [4]. In particular,

$$
\operatorname{dim}_{\mathrm{P}} X(F)=\alpha \operatorname{Dim}_{d / \alpha}^{\mathrm{FH}} F \quad \text { a.s. }
$$

Proof. Since $\left.\mathbb{E}\left[e^{i \xi \cdot X(t)}\right]=\exp \left(-\left(t_{1}+\cdots+t_{N}\right)\|\xi\|^{\alpha}\right)\right)$ for all $\xi \in \mathbb{R}^{d}$, we see that $X(t) \stackrel{d}{=} X_{1}\left(t_{1}+\cdots+t_{N}\right)$ for all $t \in \mathbb{R}_{+}^{N}$, where " $\stackrel{d}{=}$ " means equal in distribution. This and the symmetry of each $X_{j}$ together imply that

$$
\kappa_{\varepsilon}(t)=\mathbb{P}\{|\tilde{X}(t)|<\varepsilon\}=\mathbb{P}\left\{\left|X_{1}\left(\left|t_{1}\right|+\cdots+\left|t_{N}\right|\right)\right|<\varepsilon\right\} \quad \forall t \in \mathbb{R}^{N} .
$$

Since $X_{1}$ is $\alpha$-stable, it is well known that there exist constants $0<C_{1}^{\prime}<C_{2}^{\prime}<\infty$ such that uniformly for all $r>0$ and $\varepsilon \in(0,1)$,

$$
C_{1}^{\prime}\left(\frac{\varepsilon}{r^{1 / \alpha}} \wedge 1\right)^{d} \leq \mathbb{P}\left\{\left|X_{1}(r)\right|<\varepsilon\right\} \leq C_{2}^{\prime}\left(\frac{\varepsilon}{r^{1 / \alpha}} \wedge 1\right)^{d} .
$$

Then (4.3) and (4.4) imply the existence of nonrandom constants $0<C_{1}<C_{2}<\infty$ such that

$$
C_{1}\left(\frac{\varepsilon}{N^{1 / \alpha}|t|^{1 / \alpha}} \wedge 1\right)^{d} \leq \kappa_{\varepsilon}(t) \leq C_{2}\left(\frac{\varepsilon}{|t|^{1 / \alpha}} \wedge 1\right)^{d},
$$

uniformly for all $t \in \mathbb{R}^{N} \backslash\{0\}$ and $\varepsilon \in(0,1)$. It follows from this and (2.5) that

$$
\begin{aligned}
& \overline{\operatorname{dim}}_{\kappa} F \\
& =\sup \left\{\eta>0: \frac{\lim }{\varepsilon \downarrow 0} \frac{1}{\varepsilon^{\eta / \alpha}} \inf _{\mu \in \mathcal{P}(F)} \iint\left[\left(\frac{\varepsilon}{|t-s|}\right)^{d / \alpha} \wedge 1\right] \mu(d t) \mu(d s)=0\right\} .
\end{aligned}
$$


By Theorem 4.1 of Khoshnevisan and Xiao 13, we obtain

$$
\overline{\operatorname{dim}}_{\kappa} F=\alpha \mathrm{B}-\overline{\operatorname{Dim}}_{d / \alpha} F,
$$

where B-Dim $\overline{\operatorname{Dim}}_{s} F$ denotes the $s$-dimensional box dimension profiles of Howroyd [6]. By applying a similar argument as the proof of (2.40), we deduce that $\operatorname{Dim}_{\kappa} F=$ $\alpha \mathrm{P}-\operatorname{Dim}_{d / \alpha} F$, where P-Dim $s F$ denotes the $s$-dimension packing dimension profiles of Howroyd [6]. Thanks to Theorem 1.1 of [13], P-Dim ${ }_{s} F=\operatorname{Dim}_{s}^{\mathrm{FH}} F$ for all analytic sets $F \subseteq \mathbb{R}^{N}$ and $s>0$. Therefore $\operatorname{Dim}_{\kappa} F=\alpha \operatorname{Dim}_{d / \alpha}^{\mathrm{FH}} F$. Finally, (4.2) follows from Theorem 2.14

Next we use Corollary 4.1 to compute the Falconer-Howroyd packing dimension profiles of a family of random sets. In order to do so, we recall that a subordinator $\{S(t)\}_{t \geq 0}$ is a one-parameter Lévy process such that for almost all sample paths $S$ is nondecreasing and $S(t) \geq 0$ for all $t \geq 0$. The Laplace exponent $\Phi$ of a subordinator $S$ is defined by $\mathbb{E}\left[e^{-\lambda S(t)}\right]=e^{-t \Phi(\lambda)}$. Our next corollary is a direct extension of Corollary 1.3 of [9]. The proof is the same and we omit it.

Corollary 4.2. Let $S(t)=\left(S_{1}(t), \ldots, S_{N}(t)\right)$, where $S_{1}, \ldots, S_{N}$ are independent subordinators with Laplace exponents $\Phi_{1}, \ldots, \Phi_{N}$, respectively. Then for all $s \geq \frac{1}{2}$,

$$
\operatorname{Dim}_{s}^{\mathrm{FH}} S([0,1])=s(1-\theta) \quad \text { a.s., }
$$

where

$$
\theta:=\lim _{\lambda \uparrow \infty} \frac{1}{\log \lambda} \log \left(\int_{1}^{\lambda} \frac{d x}{\sum_{j=1}^{N} \Phi_{j}\left(x^{1 / s}\right)}\right) .
$$

In particular, if $S_{1}, \ldots, S_{N}$ have the same distribution, then

$$
\operatorname{Dim}_{s}^{\mathrm{FH}} S([0,1])=\operatorname{Dim}_{s}^{\mathrm{FH}} S_{1}([0,1]) \text { a.s. }
$$

Equation (4.10) is not surprising since all $N$ subordinators are driven by the same time $t$. Next we will let the subordinators driven by their own times and compute the Falconer-Howroyd packing dimension profiles again.

Corollary 4.3. Let $S(t)=\left(S_{1}\left(t_{1}\right), \ldots, S_{N}\left(t_{N}\right)\right)$ for $t \in \mathbb{R}_{+}^{N}$, where $S_{1}, \ldots, S_{N}$ are independent subordinators with Laplace exponents $\Phi_{1}, \ldots, \Phi_{N}$, respectively. Then for all $s \geq \frac{1}{2}$

$$
\operatorname{Dim}_{s}^{\mathrm{FH}} S\left([0,1]^{N}\right)=s(1-\theta) \quad \text { a.s. }
$$

where

$$
\theta:=\lim _{\lambda \uparrow \infty} \frac{1}{\log \lambda} \log \left(\int_{1}^{\lambda} \frac{d x}{\prod_{j=1}^{N} \Phi_{j}\left(x^{1 / s}\right)}\right) .
$$

In particular, if $S_{1}, \ldots, S_{N}$ have the same distribution, then

$$
\operatorname{Dim}_{s}^{\mathrm{FH}} S\left([0,1]^{N}\right) \leq N \cdot \operatorname{Dim}_{s}^{\mathrm{FH}} S_{1}([0,1]) .
$$

We can prove Corollary 4.3 by the same method as in Corollary 4.2 , provided that there is a multiparameter version of Theorem 1.1 of Khoshnevisan and Xiao [12. One way to derive the said multiparameter analog is to first find a formula for additive Lévy processes similar to that of Theorem 6 of Taylor [15] and then employ the method of [12]. However, we can also achieve this directly from Proposition 2.6, as the following shows. 
Lemma 4.4. $\operatorname{Dim}_{\kappa}[0,1]^{N}=\overline{\operatorname{dim}}_{\kappa}[0,1]^{N}$.

Proof. For every $l^{\infty}$ open ball $B(t, \varepsilon)$, define $F(t, \varepsilon):=B(t, \varepsilon) \cap[0,1]^{N}$. If $F(t, \varepsilon)$ is nonempty, then we can find finitely many points $t^{1}, \ldots, t^{n} \in \mathbb{R}^{N}$ such that $[0,1]^{N} \subseteq \bigcup_{i=1}^{n} G_{i}(t, \varepsilon)$, where $G_{i}(t, \varepsilon):=\left\{s+t^{i}: s \in F(t, \varepsilon)\right\}$. The monotonicity, finite stability, and translation invariance of $\overline{\operatorname{dim}}_{\kappa}$ together imply that

$$
\overline{\operatorname{dim}}_{\kappa} F(t, \varepsilon) \leq \overline{\operatorname{dim}}_{\kappa}[0,1]^{N} \leq \max _{1 \leq i \leq n} \overline{\operatorname{dim}}_{\kappa} G_{i}(t, \varepsilon)=\overline{\operatorname{dim}}_{\kappa} F(t, \varepsilon) .
$$

Since $B(t, \varepsilon)$ is arbitrary, we see that $\overline{\operatorname{dim}}_{\kappa}[0,1]^{N} \cap V=\overline{\operatorname{dim}}_{\kappa}[0,1]^{N}$ for every open set $V \subseteq \mathbb{R}^{N}$, provided $V \cap[0,1]^{N} \neq \emptyset$. Since $[0,1]^{N}$ is compact, an application of Baire's category theorem shows that $\operatorname{Dim}_{\kappa}[0,1]^{N}=\overline{\operatorname{dim}}_{\kappa}[0,1]^{N}$.

Lemma 4.5. Let $X$ be an $N$-parameter additive Lévy process in $\mathbb{R}^{d}$ with characteristic function $\mathbb{E}\left[e^{i \xi \cdot X(t)}\right]=\exp \left(-\sum_{j=1}^{N} t_{j} \Psi_{j}(\xi)\right)$. Furthermore, we assume that $\Psi_{j}(\xi)>0$ for all $\xi \in \mathbb{R}^{d}$ and $1 \leq j \leq N$. For each $\varepsilon>0$, define

$$
W(\varepsilon)=\int_{\mathbb{R}^{d}} \frac{\rho(\xi / \varepsilon)}{\prod_{k=1}^{d}\left(1+\xi_{k}^{2}\right)} d \xi \quad \text { with } \quad \rho(z)=\prod_{j=1}^{N} \operatorname{Re}\left(\frac{1}{1+\Psi_{j}(z)}\right) .
$$

Then

$$
\operatorname{dim}_{\mathrm{P}} X\left([0,1]^{N}\right)=\sup \left\{\eta>0: \underline{\lim }_{\varepsilon \downarrow 0} \varepsilon^{-\eta} W(\varepsilon)=0\right\}=\varlimsup_{\varepsilon \downarrow 0} \frac{\log W(\varepsilon)}{\log \varepsilon} \quad \text { a.s. }
$$

Proof. For each $\varepsilon>0$, define

$$
V(\varepsilon)=\inf _{\mu \in \mathcal{P}\left([0,1]^{N}\right)} \int_{\mathbb{R}^{d}} \frac{\mathcal{E}_{\mu}(\xi / \varepsilon)}{\prod_{k=1}^{d}\left(1+\xi_{k}^{2}\right)} d \xi,
$$

with $\mathcal{E}_{\mu}(\xi)$ defined in (2.20). Thanks to Theorem 2.14, Lemma 4.4 and Proposition 2.6. it suffices to show that there exist some nonrandom constants $0<c<C<\infty$, not depending on $\varepsilon$, such that

$$
c W(\varepsilon) \leq V(\varepsilon) \leq C W(\varepsilon) \quad \forall \varepsilon>0 .
$$

In order to show this, define for each $t \in \mathbb{R}^{N}$ and $t_{0} \in \mathbb{R}$

$$
f_{\varepsilon}\left(t, t_{0}\right):=e^{-\left|t_{0}\right|} \int_{\mathbb{R}^{d}} \exp \left(-\sum_{j=1}^{N}\left|t_{j}\right| \Psi_{j}\left(\operatorname{sgn}\left(t_{j}\right) \xi / \varepsilon\right)\right) \frac{d \xi}{\prod_{k=1}^{d}\left(1+\xi_{k}^{2}\right)} .
$$

From Fubini's theorem, we see that the Fourier transform of $f_{\varepsilon}$ satisfies

$$
\hat{f}_{\varepsilon}(z)=2^{N+1} \int_{\mathbb{R}^{d}} \operatorname{Re}\left(\frac{1}{1-i z_{0}}\right) \prod_{j=1}^{N} \operatorname{Re}\left(\frac{1}{\Psi_{j}(\xi / \varepsilon)-i z_{j}}\right) \frac{d \xi}{\prod_{k=1}^{N}\left(1+\xi_{k}^{2}\right)},
$$

for all $z \in \mathbb{R}^{1+N}$. Moreover, since $\Psi_{j}(\xi)>0$ for all $\xi \in \mathbb{R}^{d}$ and $1 \leq j \leq N, \hat{f}(z)$ is positive for all $z \in \mathbb{R}^{N+1}$. Thus we can apply Theorem 3.1 of Khoshnevisan [8] to derive that

$$
\begin{aligned}
& \inf _{\mu \in \mathcal{P}\left([0,1]^{N} \times\{0\}\right)} \iint f_{\varepsilon}\left(t-s, t_{0}-s_{0}\right) \mu\left(d t \times d t_{0}\right) \mu\left(d s \times d s_{0}\right) \\
& =\int_{[0,1]^{N}} f_{\varepsilon}(t, 0) d t=\int_{\mathbb{R}^{d}} \prod_{j=1}^{N} \frac{1-\exp \left(-\Psi_{j}(\xi / \varepsilon)\right)}{\Psi_{j}(\xi / \varepsilon)} \frac{d \xi}{\prod_{k=1}^{d}\left(1+\xi_{k}^{2}\right)} .
\end{aligned}
$$


On one hand, the elementary facts

$$
\lim _{x \rightarrow \infty} \frac{1-e^{-x}}{x} / \frac{1}{1+x}=1 \text { and } \lim _{x \downarrow 0} \frac{1-e^{-x}}{x} / \frac{1}{1+x}=1
$$

imply that there exist nonrandom constants $0<c<C<\infty$ such that $c /(1+x) \leq$ $\left(1-e^{-x}\right) / x \leq C /(1+x)$ for all $x \geq 0$. On the other hand, we have

$$
\inf _{\mu \in \mathcal{P}\left([0,1]^{N} \times\{0\}\right)} \iint f_{\varepsilon}\left(t-s, t_{0}-s_{0}\right) \mu\left(d t \times d t_{0}\right) \mu\left(d s \times d s_{0}\right)=V(\varepsilon) .
$$

Now (4.18) follows from these two facts and (4.21).

Proof of Corollary 4.3. Let $X$ be an additive Lévy process in $\mathbb{R}$, independent from $S$. Furthermore, let $X_{j}$ be isotropic stable with index $\alpha=1 / s$ for $1 \leq j \leq N$. Define $Y(t):=\sum_{j=1}^{N} X_{j}\left(S_{j}\left(t_{j}\right)\right)$ for $t \in \mathbb{R}_{+}^{N}$. Then $Y$ is an additive Lévy process in $\mathbb{R}$ with characteristic function $\mathbb{E}\left[e^{i z X(t)}\right]=\exp \left(-\sum_{j=1}^{N} t_{j} \Phi_{j}\left(|z|^{\alpha}\right)\right)$, where $\Phi_{j}$ is the Laplace exponent of $S_{j}$. On one hand, we can apply Corollary 4.1, conditionally on the process $S$, to derive

$$
\operatorname{dim}_{\mathrm{P}} X(S([0,1]))=\alpha \operatorname{Dim}_{1 / \alpha}^{\mathrm{FH}} S([0,1]) \quad \text { a.s. }
$$

On the other hand, we can apply Lemma 4.5 directly to the additive Lévy process $Y$ to derive

$$
\begin{aligned}
& \operatorname{dim}_{\mathrm{P}} X(S([0,1]))=\operatorname{dim}_{\mathrm{P}} Y([0,1]) \\
& =\varlimsup_{\varepsilon \downarrow 0} \frac{1}{\log \varepsilon} \log \left(\int_{0}^{\infty} \frac{d x}{\left(1+x^{2}\right) \prod_{j=1}^{N}\left(1+\Phi_{j}\left((x / \varepsilon)^{\alpha}\right)\right)}\right) \quad \text { a.s. }
\end{aligned}
$$

Now by applying to the last term a similar analysis of Corollary 1.3 of Khoshnevisan, Schilling, and Xiao 9], we have

$$
\begin{aligned}
& \varlimsup_{\varepsilon \downarrow 0} \frac{1}{\log \varepsilon} \log \left(\int_{0}^{\infty} \frac{d x}{\left(1+x^{2}\right) \prod_{j=1}^{N}\left(1+\Phi_{j}\left((x / \varepsilon)^{\alpha}\right)\right)}\right) \\
& =\varlimsup_{\varepsilon \downarrow 0} \frac{1}{\log \varepsilon} \log \left(\varepsilon \int_{1}^{1 / \varepsilon} \frac{d x}{\prod_{j=1}^{N} \Phi_{j}\left((x / \varepsilon)^{\alpha}\right)}\right) .
\end{aligned}
$$

It follows that

$$
\operatorname{dim}_{\mathrm{P}} X(S([0,1]))=1-\theta \quad \text { a.s. }
$$

Therefore (4.11) follows from this and (4.24).

When $S_{1}, \ldots, S_{N}$ have the same distribution, Jensen's inequality implies that

$$
\begin{aligned}
\theta & =\frac{\lim }{\lambda \uparrow \infty} \frac{1}{\log \lambda} \log \left((\lambda-1) \int_{1}^{\lambda} \frac{(\lambda-1)^{-1} d x}{\Phi\left(x^{1 / s}\right)^{N}}\right) \\
& \geq \frac{\lim }{\lambda \uparrow \infty} \frac{1}{\log \lambda} \log \left((\lambda-1)\left(\int_{1}^{\lambda} \frac{(\lambda-1)^{-1} d x}{\Phi\left(x^{1 / s}\right)}\right)^{N}\right) \\
& =1-N+N \frac{\lim }{\lambda \uparrow \infty} \frac{1}{\log \lambda} \log \left(\int_{1}^{\lambda} \frac{d x}{\Phi\left(x^{1 / s}\right)}\right) .
\end{aligned}
$$

Thus $\operatorname{Dim}_{s}^{\mathrm{FH}} S\left([0,1]^{N}\right) \leq N \cdot \operatorname{Dim}_{s}^{\mathrm{FH}} S_{1}([0,1])$. 


\section{ACKNOWLEDGEMENTS}

The author would like to thank his advisor, Professor Davar Khoshnevisan, for suggesting this problem and many useful discussions, and an anonymous referee for a very careful reading which led to corrections and improvements in the presentation.

\section{REFERENCES}

[1] Robert C. Dalang and John B. Walsh, Geography of the level sets of the Brownian sheet, Probab. Theory Related Fields 96 (1993), no. 2, 153-176, DOI 10.1007/BF01192131. MR 1227030 (94h:60055)

[2] Robert C. Dalang and John B. Walsh, The structure of a Brownian bubble, Probab. Theory Related Fields 96 (1993), no. 4, 475-501, DOI 10.1007/BF01200206. MR:1234620 (94j:60105)

[3] Kenneth Falconer, Fractal geometry, John Wiley \& Sons Ltd., Chichester, 1990. Mathematical foundations and applications. MR:1102677 (92j:28008)

[4] K. J. Falconer and J. D. Howroyd, Packing dimensions of projections and dimension profiles, Math. Proc. Cambridge Philos. Soc. 121 (1997), no. 2, 269-286, DOI 10.1017/S0305004196001375. MR1426523 (98j:28004)

[5] P. J. Fitzsimmons and Thomas S. Salisbury, Capacity and energy for multiparameter Markov processes, Ann. Inst. H. Poincaré Probab. Statist. 25 (1989), no. 3, 325-350 (English, with French summary). MR1023955 (91d:60180)

[6] J. D. Howroyd, Box and packing dimensions of projections and dimension profiles, Math. Proc. Cambridge Philos. Soc. 130 (2001), no. 1, 135-160, DOI 10.1017/S0305004100004849. MR.1797735(2002a:28010)

[7] W. S. Kendall, Contours of Brownian processes with several-dimensional times, Z. Wahrsch. Verw. Gebiete 52 (1980), no. 3, 267-276, DOI 10.1007/BF00538891. MR576887 (83a:60066)

[8] Davar Khoshnevisan, Slices of a Brownian sheet: new results and open problems, Seminar on Stochastic Analysis, Random Fields and Applications V, Progr. Probab., vol. 59, Birkhäuser, Basel, 2008, pp. 135-174, DOI 10.1007/978-3-7643-8458-6_9. MR2401955(2009m:60182)

[9] Davar Khoshnevisan, René Schilling, and Yimin Xiao, Packing dimension profiles and Lévy processes, Bull. London Math. Soc. (2012).

[10] Davar Khoshnevisan and Yimin Xiao, Level sets of additive Lévy processes, Ann. Probab. 30 (2002), no. 1, 62-100, DOI 10.1214/aop/1020107761. MR1894101(2002m:60094)

[11] Davar Khoshnevisan and Yimin Xiao, Lévy processes: capacity and Hausdorff dimension, Ann. Probab. 33 (2005), no. 3, 841-878, DOI 10.1214/009117904000001026. MR2135306 (2006d:60078)

[12] Davar Khoshnevisan and Yimin Xiao, Packing dimension of the range of a Lévy process, Proc. Amer. Math. Soc. 136 (2008), no. 7, 2597-2607, DOI 10.1090/S0002-9939-08-09163-6. MR2390532(2009d:60143)

[13] Davar Khoshnevisan and Yimin Xiao, Packing-dimension profiles and fractional Brownian motion, Math. Proc. Cambridge Philos. Soc. 145 (2008), no. 1, 205-213, DOI 10.1017/S0305004108001394. MR2431650 (2009j:60086)

[14] Davar Khoshnevisan, Yimin Xiao, and Yuquan Zhong, Measuring the range of an additive Lévy process, Ann. Probab. 31 (2003), no. 2, 1097-1141, DOI 10.1214/aop/1048516547. MR,1964960(2004c:60155)

[15] S. James Taylor, The use of packing measure in the analysis of random sets, Stochastic processes and their applications (Nagoya, 1985), Lecture Notes in Math., vol. 1203, Springer, Berlin, 1986, pp. 214-222, DOI 10.1007/BFb0076883. MR872112 (88i:60078)

[16] Ming Yang, Hausdorff dimension of the image of iterated additive processes, Rocky Mountain J. Math. 40 (2010), no. 4, 1333-1353, DOI 10.1216/RMJ-2010-40-4-1333. MR.2718817 (2012a:60143)

Department of Mathematics, University of Utah, Salt Lake City, Utah 84112 Current address: Department of Statistics and Probability, C-409 Wells Hall, Michigan State

University, East Lansing, Michigan 48824

E-mail address: lzhang81@stt.msu.edu 